\title{
Clima y satisfacción laboral en el contexto universitario
}

\author{
Vicente Pecino-Medina ${ }^{1 *}$, Miguel A. Mañas-Rodríguez ${ }^{1}$, Pedro A. Díaz-Fúnez ${ }^{1}$, Jorge López-Puga ${ }^{2}$ y \\ Juan-Manuel Llopis-Marín ${ }^{1}$ \\ 1 Universidad de Almería \\ 2 Universidad Católica San Antonio de Murcia
}

Resumen: El objetivo del estudio es comprobar la relación del clima y la
satisfacción laboral en el contexto de una universidad pública. Los datos
fueron recogidos de 318 empleados públicos del personal de administración
y servicios. Se realizaron análisis multinivel para comprobar los efectos
transnivel del clima, operacionalizado a nivel de las áreas administrativas, y
la satisfacción laboral individual. El modelo de Kopelman, Brief y Guzzo
(1990) sirve de marco teórico para mostrar las relaciones entre el clima y los
estados cognitivos y afectivos, como la satisfacción laboral. Los resultados
muestran que el clima de las áreas tiene una relación positiva y significativa
con la satisfacción laboral, lo que tiene implicaciones para el desarrollo de
prácticas innovadoras de recursos humanos que fomenten el bienestar y el
compromiso de los empleados, en la construcción de una organización sa-
ludable y responsable socialmente.
Palabras clave: Clima; satisfacción laboral; personal de administración y Palabras clave: Clima; satis
servicios; universidad pública.

\section{Introducción}

En el presente estudio comprobamos las relaciones entre el clima y la satisfacción laboral de los empleados públicos de una Universidad. Desde hace algunos años, el sistema universitario español (SUE) está siendo objeto de una profunda reflexión sobre su rendimiento, productividad y eficiencia en el cumplimiento de los objetivos y fines estratégicos recogidos en la Constitución y en sus normas de desarrollo. No es casualidad que, en apenas dos años, el Gobierno haya solicitado informes a diferentes grupos de expertos sobre la realidad actual del SUE y sus perspectivas de futuro (Informe de la Comisión de Expertos Internacionales de la EU2015 “ $A u$ dacia para llegar lejos: Universidades fuertes para la España de Manana" de 21 de septiembre de 2011; Propuestas para la Reforma y Mejora de la Calidad y Eficiencia del Sistema Universitario Europeo de 12 de febrero de 2013). En este contexto de reflexión, también ha influido la situación social, económica y política del país que ofrecen una oportunidad de consolidar los importantes logros obtenidos desde la Ley de Reforma Universitaria (1983) y, al mismo tiempo, deshacerse de los elementos que impiden una reforma en profundidad del actual SUE para adaptarse a las nuevas exigencias y demandas de la sociedad civil y a la competitividad y globalización universitarias.

Por otro lado, conocemos que las exigencias derivadas de factores psicosociales del trabajo han empeorado en los últimos años, aumentando los empleados que manifiestan problemas de salud por sufrir cansancio, agotamiento y estrés (VII Encuesta Nacional de Condiciones de Trabajo,

* Dirección para correspondencia [Correspondence address]: Vicente Pecino Medina. Universidad de Almería. Carretera de Sacramento s/n. La Cañada de San Urbano. Almería (España). E-mail: vpecino@ual.es
Title: Climate and job satisfaction in university environment.

Abstract: The aim of this paper is to determine the relationship between climate and job satisfaction in the context of a public university. Data were collected from 318 administrative and technical employees. Multilevel analyses were conducted to test the cross level effects of climate -occurring at a level of administrative areas- and individual job satisfaction. Kopelman, Brief and Guzzo's (1990) model provides a theoretical framework to show the relationships between climate and cognitive and affective states, such as job satisfaction. Results show that climate in administrative areas has a significant and possitive effect on job satisfaction, which has important implications for the development of innovative practices of human resources that encourage the welfare and commitment of employees on the building of a healthy and socially responsible organization.

Key words: Climate; job satisfaction; staff personnel; public university.
2011). Al mismo tiempo, se constata que el grado de optimismo y satisfacción de los europeos ha descendido un $20 \%$ desde que comenzó la crisis económica y más de una tercera parte de los encuestados afirman que su situación financiera se ha deteriorado en los cinco últimos años (III European Quality of Live Survey, 2012). La presente crisis económica y social añade una preocupación adicional en el ámbito de las Administraciones Públicas porque la propia institución del empleo público está siendo denostada socialmente y sometida a todo tipo de tensiones políticas y acalorados debates sobre los "privilegios" de los empleados públicos (Aunión y Sevillano, 2013). Más aún, se asiste a un empeoramiento constante de sus condiciones actuales de trabajo bajo los efectos de los recortes y ajustes presupuestarios, incluyendo despidos que, en algunos casos, ponen en riesgo su buen funcionamiento y provoca una baja motivación del personal (European Working Conditions Observatory: Working Conditions in Central Public Administration, 2013).

El personal de administración y servicios (PAS) de las Universidades Públicas conforma una burocracia profesional cuya misión es "el apoyo, asistencia y asesoramiento a las autoridades académicas, el ejercicio de la gestión y administración, particularmente en las áreas de recursos humanos, organización administrativa, asuntos económicos, informática, archivos, bibliotecas, información, servicios generales, servicios científico-técnicos, así como el soporte a la investigación y la transferencia de tecnología y a cualesquiera otros procesos de gestión administrativa y de soporte que se determine necesario para la universidad en el cumplimiento de sus objetivos" (Ley Orgánica de Universidades, artículo 73). Estos empleados públicos desempeñan puestos de trabajo adscritos y agrupados en áreas administrativas de acuerdo con la estructura diseñada en la correspondiente Relación de Puestos de Trabajo (Estatuto Básico del Empleado Público, EBEP, artículo 74) y aprobada por el Consejo de Gobierno 
de la universidad, de ahí la importancia de realizar un estudio multinivel con el clima operacionalizado a nivel de estas áreas. Sin embargo, su actividad profesional está mediatizada por una estructura de poder paralela de cargos académicos, pertenecientes al personal docente e investigador, introduciendo en la gestión universitaria un sesgo claramente "politizado" (Solé y Llinás-Audet, 2011). Diferentes estudios han mostrado que existe una fuerte relación entre la percepción de la política en las organizaciones y diferentes actitudes y conductas negativas de los empleados, como menores niveles de satisfacción y compromiso organizacional, aumento del estrés y burnout y mayores niveles de absentismo, entre otras (Ferris y Kacmar, 1992; Vigoda, 1999, 2002).

Por todo lo anterior, debemos destacar la originalidad del estudio al existir un vacío en el número de trabajos con estas características en la literatura sobre Administración Pública. Como se afirma por Vigoda-Gadot y Beeri (2012), los estudios de esta naturaleza en el sector público son raros y escasos, cuando su realización debería ser pertinente por la naturaleza del servicio a los ciudadanos que tiene la Administración Pública y por el objetivo de alcanzar un servicio público de excelencia y de calidad (EBEP, 2007). Por ello, el objetivo general de este artículo es valorar el bienestar laboral de los empleados de una universidad pública a través de las relaciones entre clima y satisfacción laboral.

\section{El estudio del clima organizacional}

La investigación del clima cobra sentido por el interés en conocer y comprender el comportamiento de los individuos en el contexto laboral, en entender las formas en que los miembros de una organización experimentan, sienten, esa organización (Schneider, 2000). Desde los primeros trabajos de investigación (Lewin, Lippitt y White, 1939), el clima se ha convertido en uno de los tópicos más influyentes en la investigación del comportamiento organizacional. No obstante, cuando intentamos definirlo, nuestra primera impresión es de cierta confusión y ambigüedad, así Schneider (1990) indicó que dicho reto era como intentar "clavar gelatina en la pared". A pesar de ello, se adoptará una de las definiciones más extendidas en la literatura sobre clima, la propuesta por Schneider y colegas (Schneider, Ehrhart y Macey, 2011) que definen el clima como el conjunto de percepciones compartidas relacionadas con las políticas, prácticas y procesos así como las conductas que una organización recompensa, apoya y espera en un contexto laboral y el significado psicológico que ellas adquieren para sus miembros.

De esta definición extraemos dos notas de interés (Kuenzi y Schminke, 2009). En primer lugar, el clima es un constructo perceptual más que una característica objetiva de la organización (James y Jones, 1974; Schneider, 2000), por lo que es conceptualizado como conjuntos de descripciones que se basan en las percepciones de las características, eventos y procesos organizacionales (Ostroff, Kinicki y Tamkins, 2003). Los individuos no responden directamente al contexto laboral sino que antes perciben e interpretan ese contexto
(Carr, Schmidt, Ford y DeShon, 2003), adquiriendo significado psicológico para cada uno de ellos (James et al., 2008). En segundo lugar, el clima es un fenómeno colectivo. Sus orígenes descansan en las percepciones individuales, pero es una propiedad de la organización. Así, el clima operacionalizado a niveles superiores de análisis (equipos, áreas, etc.) se suele definir como las percepciones compartidas por los miembros de ese equipo o unidad de nivel superior (Schneider, Ehrhart y Macey, 2013).

La evolución del estudio del clima organizacional no ha estado exenta de polémicas y dilemas, entre ellas, la fundamentación teórica, la dimensionalidad del clima y los asuntos metodológicos relacionados con la agregación de las percepciones individuales. Sin embargo, pese a estas dificultades, su estudio ha proporcionado una "alternativa necesaria a las teorías de la motivación para todo lo que ocurre a las personas en el contexto laboral' (Schneider y Reichers, 1983; p.20). Este progreso se ha mostrado en evidencias sobre sus antecedentes, efectos y factores que influyen en la eficiencia organizacional, en el diseño de intervenciones para mejorar las prácticas y procedimientos de los sistemas estratégicos de recursos humanos (Bowen y Ostroff, 2004; James et al., 2008) y en la superación de los dilemas (Bliese, 2000; Carr et al., 2003; Klein et al., 2000; Schneider et al., 2011). De igual manera, la evolución del estudio del clima condujo a los investigadores desde su consideración como un concepto global o molar, con la presunción de que los individuos desarrollan percepciones globales o sumarias de su organización (James y Jones, 1974; Schneider y Bartlett, 1968), hasta la emergencia de los climas estratégicos y específicos centrados en la consecución de metas relevantes para la organización (Schneider, 1975). En la actualidad, la investigación está retomando el estudio de los climas globales, conceptualizándolos como fuentes de los climas estratégicos y específicos (Kuenzi y Schminke, 2009; Schneider et al., 2011) e incluyéndose en diferentes marcos teóricos y tendencias actuales preocupadas en la consecución del bienestar psicosocial de los empleados (p.e., psicología positiva organizacional) y en la construcción de organizaciones más saludables (Luthans, Norman, Avolio y Avey, 2008; Salanova, Llorens, Cifre y Martinez, 2012).

En este sentido, la formación del clima se fundamenta en los procesos de interacción social de los miembros de los equipos, unidades u organizaciones. Esta aproximación basada en el interaccionismo simbólico (Blumer, 1969; Schneider y Reichers, 1983), propone que la similitud en las percepciones de clima de los empleados se desarrolla a partir de las interacciones entre ellos (Reichers y Schneider, 1990). A través de la interacción social, los empleados comparten los significados que atribuyen a los eventos y prácticas organizacionales, construyendo socialmente el clima. Además, la investigación ha mostrado que cuando las personas trabajan juntas, pueden compartir creencias y experiencias afectivas y, así, muestran unos patrones motivacionales y conductuales similares (González-Romá, Peiró, Subirats y Mañas, 2000). Los miembros de los grupos dentro de las organizaciones tienden a experimentar las mismas políticas, decisiones y 
efectos, lo que previsiblemente conduzca a la emergencia del clima en esas unidades de trabajo, en nuestro caso, las áreas administrativas en las que se estructura la administración universitaria (Howe, 1977; Schneider, 1975). Del mismo modo, el modelo del procesamiento social de la información formulado por Salancik y Pfeffer (1978) y la teoría social cognitiva de Bandura (2001) nos explican cómo los empleados intercambian opiniones sobre los acontecimientos importantes de la organización (políticas, decisiones, etc.) con otros miembros de los grupos o unidades, alcanzando consensos en su interpretación de dichos acontecimientos para poder comprender sus propias experiencias organizacionales. Así, los empleados modelan sus propias conductas a través de la observación de los estímulos contextuales, especialmente de las conductas de otros empleados (jefes y compañeros), e identifican indicios sociales sobre conductas y normas que son deseables en la organización. En este proceso de interacción continuo y repetitivo, el clima se expande y se refuerza en toda la organización y en sus áreas administrativas (Craig-Wallace et al, 2011; Dragoni, 2005). Este, sin lugar a dudas, es un aspecto relevante en la conceptualización del clima: proporcionar evidencia de que las percepciones de clima a nivel individual emergen hasta convertirse en un fenómeno a nivel de unidad o de área administrativa (Kozlowski y Klein, 2000; Morgeson y Hoffman, 1999).

Por estas consideraciones, el presente trabajo se centra en el estudio del clima agregado a nivel de las áreas que conforman la administración universitaria siguiendo una tendencia consolidada desde los inicios de la investigación sobre clima (Glick, 1985; Hellriegel y Slocum, 1974) hasta los estudios más recientes sobre climas específicos y de procesos (Ambrose, Schminke y Mayer, 2013; Martinez-Tur, Tordera, Peiró y Potocnik, 2011; Van de Voorde, Van Veldhoven y Paauwe, 2012).

\section{Clima y Satisfacción laboral}

Sin lugar a dudas, la satisfacción laboral es el tópico de carácter positivo más investigado en la historia de la Psicología Industrial y Organizacional (Judge y Klinger, 2007; Wright, 2006); además, ocupa un papel central en muchas teorías y modelos sobre las actitudes y conductas de los individuos, debido a sus aplicaciones prácticas en la mejora de las vidas de los empleados y en la búsqueda de la "felicidad" (Wright, 2005) y en el logro de la eficiencia organizacional (Bowen y Ostroff, 2004; Ostroff y Bowen, 2000). La definición más utilizada y difundida de la satisfacción laboral es la ofrecida por Locke (1976) que la define como "estado emocional positivo o placentero resultante de la percepción subjetiva de las experiencias laborales de la persona" (p.1300). Desde esta perspectiva, la satisfacción laboral es una actitud o conjunto de actitudes que un empleado tiene hacia su trabajo y los factores con él relacionados, trataríamos con una actitud fruto de actitudes específicas relacionadas con aspectos del trabajo y de la organización (Peiró, 1984). No obstante, las últimas ten- dencias en el estudio de la satisfacción laboral conectadas con la "revolución afectiva" (Barsade, Brief y Spataro, 2003), señalan que la conducta es una consecuencia, más que un componente de las actitudes, por lo que éstas se concretarían en dos únicos componentes: cognitivo y afectivo. Así, Judge, Hulin y Dalal (2012) definen las "satisfacciones laborales" como respuestas psicológicas multidimensionales hacia el trabajo, incluyendo componentes cognitivos (evaluativos) y afectivos (emocionales). La definición en plural de estos autores intenta explicar que no sólo es significativo considerar la satisfacción laboral en un sentido global o general, sino también es significativo considerar las satisfacciones con aspectos más concretos de nuestro trabajo (compañeros, retribución, empresa, etc.).

Para finalizar, las profundas transformaciones que se han producido en las organizaciones y en el trabajo, además del efecto que la crisis económica actual proyecta sobre el mundo laboral con un alarmante desempleo, aconsejan una mirada hacia la conservación de niveles adecuados de "felicidad" en los empleados. Esta preocupación se manifiesta en este trabajo en el estudio de la satisfacción laboral, como un elemento esencial del "santo grial" de la investigación organizacional: la tesis del "feliz y productivo empleado" (Weiss, 2002; Wright, 2005).

En la revisión de la literatura realizada se han encontrado diferentes modelos conceptuales que explican las conexiones entre el clima y sus consecuentes, en especial la productividad y la eficiencia organizacional, así como los procesos psicológicos que subyacen a esas conexiones (Carr et al., 2003; Ferris et al., 1998; Kopelman, Brief y Guzzo, 1990; Ostroff y Bowen, 2000). Dado que los modelos de Ferris et al. (1998), Ostroff y Bowen (2000) y Carr et al. (2003) se fundamentan en el modelo presentado por Kopelman et al. (1990) (véase Figura 1), para el objetivo de este estudio nos centraremos en este último.

Kopelman et al. (1990) mostraron una preocupación acerca de cómo el contexto laboral (cultura y clima) contribuye a la eficiencia organizacional (productividad). A partir de esta idea desarrollaron un modelo que conecta la cultura organizacional con la productividad a través de diferentes elementos claramente diferenciados (véase Figura 1). La cultura organizacional tiene una notable influencia en el tipo de prácticas de gestión de recursos humanos que una organización implementa para conseguir una mayor productividad y eficiencia. Y ese impacto sobre la productividad se logra a través de sus efectos sobre el clima organizacional, esto es, sobre cómo los empleados interpretan su contexto y entorno laboral. Por tanto, el clima tiene un papel mediador entre las prácticas de recursos humanos y la productividad. A su vez, el clima se traduce en importantes conductas de los empleados ligadas al desempeño, al logro y a la ciudadanía, todas ellas relacionadas directamente con la productividad. Esta conexión se realiza a través de determinados estados cognitivos y afectivos organizacional, en concreto la motivación y la satisfacción laboral. 


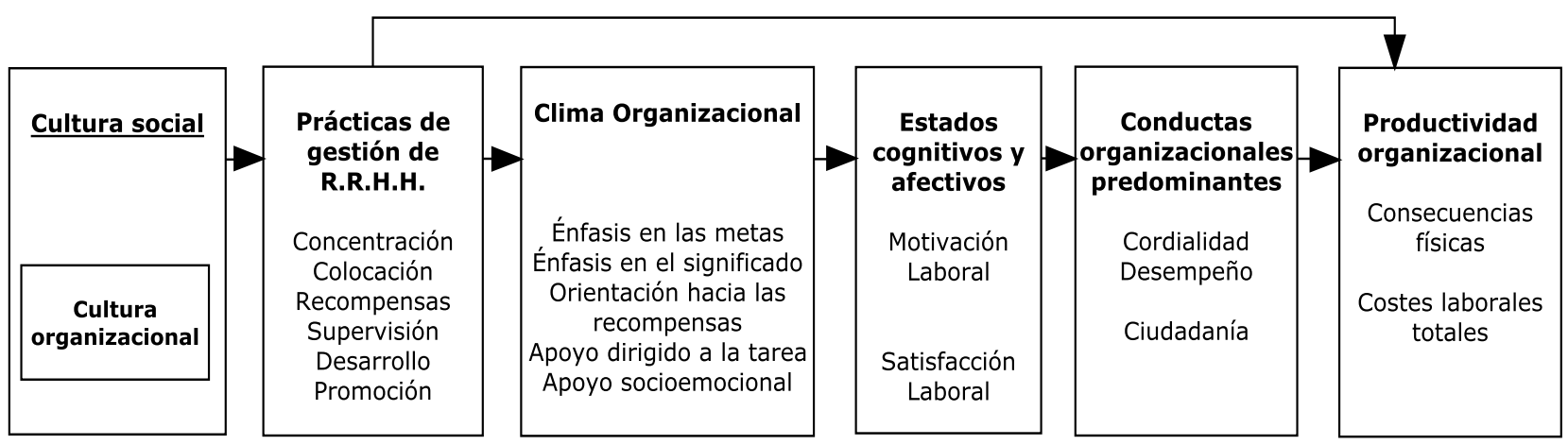

Figura 1. Modelo de Kopelman, Brief y Guzzo (1990).

Por tanto, existen dos elementos relevantes del modelo de Kopelman et al. (1990) que son importantes para nuestro estudio. Por un lado, el clima a nivel de las áreas administrativas en las que se estructura la administración universitaria y los estados cognitivos y afectivos de los empleados, entre los que incluimos la satisfacción laboral. En relación con ellos existen numerosos estudios que han confirmado sus relaciones significativas en diferentes contextos ocupacionales, mostrando que cuando los empleados perciben más positivamente los climas de los que forman parte se sienten más satisfechos y, en consecuencia, las organizaciones muestran índices de mayor productividad y desempeño (Mañas, González-Romá y Peiró,1999; Medina, Munduate, Martinez, Dorado y Mañas, 2004; Ostroff, 1992, 1993; Patterson, Warr y West, 2004; Schneider, 1990; Schneider, White y Paul, 1998; Schulte, Ostroff y Kinicki, 2006).

Por cuanto antecede, formulamos la siguiente hipótesis:

Hipótesis: El clima de las áreas se relacionará positiva y significativamente con la satisfacción laboral individual.

\section{Método}

\section{Participantes y procedimiento}

La muestra está compuesta por 482 empleados públicos de administración y servicios de una Universidad Pública, agrupados en 18 áreas administrativas. En diciembre de 2008, la Gerencia de la Universidad envío a todos los empleados públicos un cuestionario on-line sobre clima y satisfacción laboral, respondieron 442 empleados, aunque sólo resultaron válidos un total de 318 cuestionarios, lo que representa el $65.97 \%$ de participación.

Asimismo, los participantes se distribuyen en función de las siguientes variables. En la variable "Edad" el colectivo de "36 a 45 años" es el más numeroso, con un $60.38 \%$. En la variable "Sexo" el 51.26\% son mujeres. En la variable "Nivel de Estudios" los participantes con estudios universitarios alcanzan el $57.86 \%$.

\section{Instrumentos}

El clima ha sido medido mediante el cuestionario FOCUS-93 (González-Romá, Tomás y Ferreres, 1995; Van Muijen et al., 1999). La escala utilizada para la evaluación de esta variable estaba compuesta por 40 ítems (p.e., "Cuánta gente que desea progresar obtiene apoyo de sus superiores"). Para todos los ítems, las respuestas presentaban un formato tipo Likert de 6 puntos, desde "1: Nadie / Nunca" a "6: Todos / Siempre".

La satisfacción laboral fue medida mediante el cuestionario CSLPS-EAP/33 (Lloret, González-Romá y Peiró, 1993), adaptado a la muestra específica del personal de administración y servicios de la Universidad. La escala utilizada para la evaluación de esta variable estaba compuesta por 33 ítems (p.e., "El reconocimiento de su trabajo por parte de los otros miembros de su servicio/unidad"). La escala de respuesta de los ítems oscila entre "1: Muy Insatisfecho" y "7: Muy Satisfecho".

\section{Análisis Estadísticos}

El objetivo del presente estudio requiere utilizar diferentes análisis estadísticos. En primer lugar, al tratarse de un modelo transnivel donde la variable clima es agregada a nivel de áreas de la administración universitaria, se requiere la adecuada justificación estadística para la agregación de datos de la variable clima. En segundo lugar, debemos realizar un análisis de correlación entre las variables para comprobar si su relación es significativa o no. Por último, para comprobar la relación transnivel de ambas variables se utilizaron los modelos de análisis de varianza de un factor de efectos aleatorios $(A E A)$ y de regresión de medias como resultados (RMR) (Pardo, Ruiz y San Martín, 2007; Raudenbush y Bryk, 2002).

\section{Índices de Agregación}

Para apoyar la agregación de las puntuaciones de clima desde un nivel individual al nivel de las áreas de la administración universitaria, deben realizarse una serie de comprobaciones estadísticas. Por tanto, aunque los datos obtenidos 
del clima fueron medidos a nivel individual, se asume la existencia de un proceso "bottom-up" en el que las propiedades al nivel inferior emergen para conformar fenómenos colectivos (Kozlowski y Klein, 2000). Estos procesos adoptan un modelo de composición, donde las relaciones entre los constructos deben ser especificadas en los diferentes niveles de análisis (Chan, 1998). De acuerdo con este autor, dentro de la tipología de los modelos de composición, los modelos de consenso directo requieren un acuerdo intra-grupo que supone que los miembros de las áreas componen grupos homogéneos, compartiendo percepciones, conductas, intereses y otras características (Klein, Conn, Smith y Sorra, 2001). En consecuencia, las decisiones para utilizar medidas agregadas de las variables deben ser acompañadas de justificación estadística (Klein, Dansereau y Hall, 1994).

En el presente trabajo se han utilizado diferentes índices estadísticos para justificar la agregación al nivel superior de análisis. En concreto, el índice de acuerdo interjueces $\left(r_{w g}\right)$; James, Demaree y Wolf, 1984), el índice de desviación promedio $\left(A D_{\mathrm{M}(\mathrm{J})}\right)$ (Burke, Finkelstein y Dusig, 1999) y los coeficientes de correlación intraclase (ICC1 e ICC2) (Bliese, 2000).

\section{Resultados}

El primer paso en el análisis de datos fue comprobar la validez de la variable clima para justificar la agregación de las puntuaciones individuales a un nivel superior de análisis: homogeneidad intra-grupo y variación entre grupos. Para valorar el acuerdo intra-grupo, fue utilizado el índice $n_{\mathrm{WG}(\mathrm{I})}$ (James, Demaree y Wolf, 1984) obteniéndose un valor medio de: $n_{\mathrm{WG}(1)}=.59$. Este resultado es similar a los obtenidos en otros estudios (p.e., Chun, Shin, Choi y Kim, 2013), indicando que las percepciones del clima fueron suficientemente homogéneas para la agregación intra-grupo (Bliese, 2000; Dunlap, Burke y Smith-Crowe, 2003; LeBreton y Senter, 2008). También se calculó el índice de desviación promedio $\left(A D_{\mathrm{M}(\mathrm{I})}\right)$ como índice de acuerdo intra-grupo, resultando un valor medio de $A D_{\mathrm{M}(\mathrm{J})}=.90$ (cut-off c/ $6=1.01$ ), mostrando una alta homogeneidad en la percepción de clima (Dunlap, Burke y Smith-Crowe, 2003).

Por último, el acuerdo intra-grupo también puede ser comprobado mediante el cálculo de los índices de correlación intraclase (ICC1 e ICC2) (James, 1982; Shrout y Fleiss, 1979). Los resultados para ambos índices fueron: ICC(1)= .27 y $\operatorname{ICC}(2)=.65$, respectivamente. Ambos valores pueden ser considerados aceptables si son comparados con otros índices observados en la revisión de la literatura (p.e., Sora, Caballer, Peiró y Witte, 2009). Los resultados obtenidos en los índices y coeficientes citados muestran valores aceptables para la agregación a nivel de áreas de la administración universitaria, al existir una homogeneidad intra-grupo y una varianza entre grupos suficiente (LeBreton y Senter, 2008).

El segundo paso en el análisis de los datos, fue la obtención de los datos descriptivos, la fiabilidad de las escalas utilizadas y el índice de correlación bivariada. En primer lugar, la media de las puntuaciones es de 3.25 para clima y de 4.28 para satisfacción laboral. La fiabilidad obtenida de las escalas es de $\alpha=.95$ para clima y de $\alpha=.97$ para satisfacción laboral. Por último, el índice de correlación bivariada fue de $r=.693$ $(p<.001)$, que indica una alta correlación entre las variables.

En el tercer paso, para confirmar las relaciones del clima (a nivel de áreas administrativas) y la satisfacción laboral (a nivel de empleados), se estimó un modelo jerárquico (Raudenbusch y Bryk, 2002) o multinivel (Goldstein, 2003) utilizando el procedimiento MIXED de la aplicación IBM SPSS 21 (Pardo, Ruiz y San Martín, 2007). A continuación, se exponen los resultados obtenidos en este procedimiento aplicando los modelos de análisis de varianza de un factor de efectos aleatorios $(A E A)$ y de regresión de medias como resultados (RMR) (Raudenbush y Bryk, 2002).

En las Tablas 1 y 2 se muestran los resultados de ambos modelos ( $A E A$ y $R M R$ ). La Tabla 1 (Modelo $A E A$ ) recoge el valor estimado de la intersección, se trata de una estimación de la media de las áreas en satisfacción laboral.

Tabla 1. Parámetros de efectos fijos.

\begin{tabular}{c|c|c|c|c|c|c|}
\hline Modelo & Parámetro & Estimación & Error Típico & $\boldsymbol{g} \boldsymbol{l}$ & $\boldsymbol{t}$ & $\boldsymbol{S i g}$ \\
\hline \multirow{2}{*}{$\boldsymbol{E A}$} & Intersección & 4.269 & .080 & 10,671 & 53.423 & .001 \\
\hline \multirow{2}{*}{$\boldsymbol{R} \boldsymbol{R} \boldsymbol{R}$} & Intersección & 4.251 & .070 & 16,311 & 60.876 & .001 \\
& Clima & 1.041 & .059 & 315,625 & 17.674 & .001 \\
\hline
\end{tabular}

$A E A$ : análisis de varianza de un factor de efectos aleatorios; $R M R$ : análisis de regresión de medias como resultados

En la Tabla 2 (Modelo $A E A$ ) se muestran las estimaciones de los parámetros de covarianza, es decir, las estimaciones de los parámetros asociados a los efectos aleatorios del modelo.

Tabla 2. Parámetros de covarianza.

\begin{tabular}{|c|c|c|c|c|c|c|}
\hline \multicolumn{2}{l|}{ Modelo Parámetro } & \multicolumn{3}{|c|}{ Estimación Error Típico Wald Z } & Sig \\
\hline \multirow{2}{*}{ AEA } & Residuos & varianza & 1.148 & .094 & 12.208 & .000 \\
\cline { 2 - 8 } & Área & & .039 & .043 & .907 & .364 \\
\hline \multirow{2}{*}{ RMR } & Residuos & varianza & .569 & .046 & 12.271 & .000 \\
\cline { 2 - 7 } & Área & & .047 & .028 & 1.637 & .102 \\
\hline
\end{tabular}

$A E A$ : análisis de varianza de un factor de efectos aleatorios; RMR: análisis de regresión de medias como resultados.

Según estas estimaciones, la variabilidad en la satisfacción laboral entre las Áreas representa el 3.26\% (ICC=.0326) (Bliese, 2000; p.358) de la variabilidad total, esto es, de las diferencias entre las medias de las áreas.

En la Tabla 1 (Modelo RMR) se muestran las estimaciones de los dos parámetros de efectos fijos, es decir, de la intersección $(\gamma=4.251)$ y del coeficiente asociado a la covariable clima $(\gamma=1.041)$. Asimismo, en la Tabla 2 se muestran las estimaciones de los parámetros de covarianza. Se comprueba que la estimación de la varianza de los residuos $(\sigma$ $=.569$ ) es inferior a la obtenida en el modelo nulo ( $\sigma=$ $1.148)$; por tanto, la variabilidad en la satisfacción laboral pa- 
rece estar en parte explicada por el clima. Además, comparando las estimaciones de los parámetros de covarianza del modelo nulo $(A E A)$ y del modelo que incluye clima ( $R M R$ ) puede conocerse la proporción de varianza explicada en la satisfacción de los empleados: .4811. En conjunto, estos resultados confirman nuestra hipótesis que predice que el clima está positiva y significativamente relacionado con la satisfacción laboral de los empleados.

Para finalizar, se compararon estadísticos de ajuste global chi-cuadrado $\left(\chi^{2}\right)$ que nos indican en qué medida el modelo propuesto es capaz de representar la variabilidad observada en los datos. Cuando comparamos ambos estadísticos (McCullag y Nelder, 1989), se comprueba que la varianza entre las áreas es distinta de cero. En concreto, se obtuvo $\chi^{2}=956.466$ con el modelo nulo y al incluir en el modelo la covariable clima se obtuvo $\chi^{2}=743.321$, la diferencia entre ambos valores $(\Delta \chi 2=214.694, p=.003)$. Por tanto, este último modelo (RMR) ajusta mejor a los datos que el modelo inicial $(A E A)$, lo que apoya la inclusión en el modelo de la variable clima.

\section{Discusión/Conclusiones}

El presente estudio contribuye al conocimiento sobre el clima organizacional en diferentes aspectos. En primer lugar, se demuestra que los empleados públicos desarrollan percepciones homogéneas en relación con el clima de las áreas de la administración universitaria (homogeneidad intragrupo) y, además, se comprueba que esas percepciones de los empleados públicos varían entre las diferentes áreas, lo que implica la existencia de diferentes valoraciones del clima (varianza entre grupos). Estos resultados confirman que el clima es un constructo colectivo (Morgeson y Hoffman, 1999; Kuenzi y Schminke, 2009) en el que los estímulos contextuales sirven como fuentes de información que indican la conveniencia o no de determinadas actitudes y conductas de los empleados. De esta forma, a través de los procesos de interacción entre los empleados y del procesamiento social de la información, el clima emerge en las áreas administrativas estudiadas influyendo sobre los empleados que pertenecen a ellas (Craig-Wallace et al., 2011). Al mismo tiempo, las diferencias existentes entre las percepciones que los empleados de las diferentes áreas administrativas tienen del clima confirman los resultados obtenidos en diferentes estudios (p.e., Howe, 1977; Ambrose, Schminke y Mayer, 2013; Van de Voorde, Van Veldhoven y Paauwe, 2012), porque las áreas son estructuras organizacionales delimitadas no sólo orgánica y formalmente sino también espacialmente en diferentes localizaciones, así los empleados que se encuentran en diferentes espacios organizacionales tienden a percibir de forma diferente las experiencias relacionadas con los contextos laborales, esto es, las políticas, prácticas y procedimientos organizacionales que se implementan en la gestión de los recursos humanos.

En segundo lugar, el clima de las áreas administrativas fue elaborado a partir de las respuestas individuales de los empleados utilizando un modelo de composición de consenso directo. La composición significativa del clima a nivel de las áreas requiere: un apropiado modelo de composición, una consistencia intra-áreas en las respuestas a nivel individual y unas diferencias inter-áreas en dichas respuestas (Chan, 1998). Los resultados obtenidos confirman todas las exigencias necesarias para este tipo de modelos.

En tercer lugar, el análisis de modelos lineales jerárquicos proporciona estimaciones de la relación transnivel que conecta el clima de las áreas administrativas con la satisfacción laboral de los empleados de dichas áreas y, asimismo, aporta valor al presente estudio (Ostroff, 1993). Los resultados obtenidos muestran que clima y satisfacción laboral están significativa y positivamente relacionados $(r=.693 ; p<.001)$, resultado que confirma los obtenidos en otros muchos estudios (p.e., Mañas et al., 1999; Ostroff, 1993; Patterson et al., 2004; Schulte et al., 2006). Y por último, se demuestra que las puntuaciones de clima agregadas a nivel de área/servicio predicen la varianza en las puntuaciones de satisfacción laboral a nivel individual, esto es, el $48.11 \%$ de las diferencias individuales observadas en la satisfacción laboral a través de las áreas de la administración universitaria son diferencias atribuibles al clima de esas áreas. Esas percepciones del clima tienen una importancia capital al proporcionar una conexión entre las políticas, prácticas y procedimientos de gestión de recursos humanos y la satisfacción laboral, actitud ligada significativa y positivamente con el compromiso (p.e., González-Romá et al., 2000) y las conductas de ciudadanía organizacional (Organ y Ryan, 1995).

Por último, el modelo formulado por Kopelman et al. (1990) explica cómo el contexto organizacional, caracterizado por la cultura y el clima, contribuye a la eficiencia organizacional que se muestra en términos de productividad. La imposibilidad de una confirmación de todos los elementos del modelo en un único estudio, aconseja la confirmación parcial de los diferentes elementos, tal como se hace en el presente estudio. Los resultados obtenidos confirman que el clima está conectado significativamente con estados afectivos y cognitivos de los empleados, tal como queda demostrado respecto a la relación positiva con la satisfacción laboral. La relevancia del clima se centra en sus efectos sobre conductas consideradas importantes para el desempeño y la eficiencia organizacional (Pirola-Merlo et al., 2002) y esa influencia se realiza a través de los estados afectivos y cognitivos de los empleados.

Los resultados obtenidos en el presente estudio tienen importantes implicaciones para la dirección universitaria en la gestión del personal de administración y servicios de las universidades públicas. En primer lugar, la dirección universitaria debería cuestionarse qué factores contribuyen a la satisfacción laboral de los empleados, conociendo que aquellas organizaciones que incrementan la satisfacción laboral puedan ser más eficientes y eficaces en el cumplimiento de sus objetivos estratégicos e institucionales (Ostroff, 1992). Por eso, es relevante considerar en este ámbito público cuál es el papel de los directivos de las áreas administrativas en la de- 
terminación del clima. Los líderes actúan como filtros al comunicar a los empleados qué características de la organización deben ser atendidas por ser estratégicas e importantes (Schein, 2004) y, en consecuencia, dependiendo del tipo de liderazgo pueden emerger diferentes climas (Lewin, Lippit y White, 1939). De este modo, la existencia de programas de desarrollo profesional de las competencias de estos empleados adquiere una dimensión estratégica singular, centrada en el cambio hacia patrones de conductas transformadoras de las organizaciones públicas (Judge y Piccolo, 2004).

En segundo lugar, la preocupación de la dirección universitaria por el desempeño individual y la eficiencia organizacional aconsejaría la realización de estudios de clima y satisfacción como parte de sus esfuerzos por diagnosticar y mejorar la administración universitaria en estos turbulentos tiempos de cambios en el empleo público. Parker et al. (2003) manifestaron que las evaluaciones del clima laboral deberían ser parte de las intervenciones que intentan mejorar la calidad de la vida laboral y el bienestar de los empleados. Asimismo, Ferris et al. (1998) afirmaron que la valoración de la eficiencia organizacional necesita utilizar múltiples criterios de evaluación. Entre esos criterios se encuentran los resultados de recursos humanos, que incluyen la satisfacción laboral de los empleados como indicador significativo. Sin duda, esa eficiencia organizacional se manifiesta en el desempeño laboral, tanto relacionado con las tareas del puesto (Brief y Motowidlo, 1986; Borman y Motowidlo, 1993) como con el desempeño contextual (altruismo, ayuda, ciudadanía) (Organ, 1988). La conexión y efectos entre satisfacción laboral, rendimiento y eficiencia organizacional han sido mostradas en numerosos trabajos (p.e., Judge, Thoresen, Bono y Patton, 2001).

No obstante, nuestros resultados deben ser considerados bajo las siguientes limitaciones. Primera, los resultados se han obtenido a partir de cuestionarios on-line cumplimentados por los empleados (auto-informes), por lo que podrían estar afectados por la varianza del método común (Avolio, Yammarino y Bass, 1991). Segunda, la muestra es muy específica, se circunscribe al colectivo del personal de adminis-

\section{Referencias}

Ambrose, M. L., Schminke, M. y Mayer, D.M. (2013). Trickle-Down Effects of Supervisor Perceptions of Interactional Justice: A Moderated Mediated Approach. Journal of Applied Psychology, 98, 678-689.

Aunión, J. A. y Sevillano, E. G. (2013, 10 de diciembre). ¿Quién debe ser funcionario? El País. Recuperado de http://www.sociedad.elpais.com/sociedad/2013/12/10/actualidad/

Avey, J. B., Reichard, R. J., Luthans, F. y Mhatre, K. H. (2011).Meta-analysis of the Impact of Positive Psychological Capital on Employee Attitudes, Behaviors, and Peformance. Human Resource Development Quarterly, 22, 127-152.

Avolio, B. J., Yammarino, F. J. y Bass, B. M. (1991). Identifying Common Method Variance with Data collected from a Single Course: An Unresolved sticky Issue. Journal of Management, 17, 571-587.

Bandura, A. (2001). Social Cognitive Theory: An Agentic Perspective. Annual Review of Psychology, 52, 1-26.

Barsade, S. G., Brief, A. P. y Spataro, S. E. (2003). The Affective Revolution in Organizational Behavior: The Emergence of a Paradigm. En J. tración y servicios de una Universidad pública, por lo que no se pueden generalizar sus resultados a otro tipo de organizaciones. Tercera, el diseño de investigación es de naturaleza transversal, lo que nos impide extraer conclusiones sobre el orden temporal de los efectos y las relaciones de causalidad.

A pesar de las limitaciones indicadas, se pueden avanzar futuras líneas de investigación. Primera, se hace necesario realizar estudios longitudinales que nos permitan analizar la evolución e influencias causales del clima sobre la satisfacción laboral en las organizaciones (Jackofsky y Slocum, 1988) y viceversa, esto es, la posibilidad de relaciones recíprocas entre clima y satisfacción laboral (James et al., 2008; Mañas, González-Romá, Peiró y Lloret, 2003; Salanova, Agut y Peiró, 2005). Segunda, sería recomendable introducir el análisis y el estudio simultaneo de múltiples climas o de configuraciones de clima (Carr et al., 2003; González-Romá, 2011; Schulte, Ostroff, Shmulyian y Kinicki, 2009). De especial interés sería el estudio del clima de servicio (Schneider, White y Paul, 1998; Schneider, Salvaggio y Subirats, 2002), como elemento clave de evaluación de una Administración Pública por sus clientes. Por último, también sería importante profundizar en el estudio de los antecedentes y consecuentes de la satisfacción laboral prestando atención a las aproximaciones disposicionales (Judge, Hulin y Dalal, 2012), al impacto del capital psicológico positivo (Avey, Reichard, Luthans y Mhatre, 2011) y a las conductas relacionadas con ella (ciudadanía organizacional, conductas contraproductivas, absentismo, etc.).

En conclusión, este estudio se ha centrado en un contexto laboral específico como es la administración universitaria. No se han encontrado en la literatura, estudios de esta naturaleza, en especial por la ausencia de iniciativas de investigación sobre el colectivo de administración y servicios universitario. En general, un sector público poco proclive y reacio a la realización de trabajos que pudieran directa o indirectamente evaluar el impacto de las políticas adoptadas por la dirección universitaria y sus efectos en las actitudes y conductas de los empleados públicos.

Greenberg (Ed)., Organizational Behavior: The State of Science (pp.3-52). New Jersey, NJ: Lawrence Erlbaum.

Bliese, P. D. (2000). Within-Group Agreement, Non-Independence, and Reliability: Implications for Data Aggregation and Analysis. En K. J. Klein y S.W.J. Kozlowski (Eds.), Multilevel Theory, Research, and Methods in Organizations (pp.349-381). San Francisco, CA: Jossey-Bass.

Blumer, H. (1969). The Methodological Position of Symbolic Interactionism. Symbolic interactionism: Perspective and Method, 1-60.

Borman, W. C. y Motowidlo, S. M. (1993). Expanding the Criterion Domain to include Elements of Contextual Performance. En N. Schmitt y W. C. Borman (Eds.), Personnel Selection in Organizations (pp. 71-98). San Francisco, CA: Jossey-Bass

Bowen, D. E. y Ostroff, C. (2004). Understanding HRM-Firm Performance linkages: The Role of the "Strenght" of the HRM System. Academy of Management Review, 29, 203-221.

Brief, A.P. y Motowidlo, S.J. (1986). Prosocial Organizational Behaviors. Academy of Management Review, 11, 710-725. 
Burke, M. J., Finkelstein, L. M. y Dusig, M. S. (1999). On Average Deviaton Indices for Estimating Interrater Agreement. Organizational Research Methods, 12, 49-68.

Carr, J. Z., Schmidt, A. M., Ford, J. K. y DeShon, R. P. (2003). Climate Perceptions Matter: A Meta-Analytic Path Analysis relating Molar Climate, Cognitive and Affective States and Individual Level Work Outcomes. Journal of Applied Psychology, 88, 605-619.

Chan, D. (1998). Functional Relations among Constructs in the Same Content Domain at Different Levels of Analysis: A Typology of Composition Models. Journal of Applied Psychology, 83, 234-246.

Chun, J. S., Shin, Y., Choi, J. N. y Kim, M. S. (2013). How does Corporate Ethics contribute to Firm Financial Performance? The Mediating Role of Collective Organizational Commitment and Organizational Citizenship Behavior. Journal of Management, 39, 853-877.

Craig-Wallace, J., Johnson, P. D., Mathe, K. y Paul, J. (2011). Structural and Psychological Empowerment Climates, Performance, and the Moderating Role of Shared Felt Accountability: A Managerial Perspective. Journal of Management, 96, 840-850.

Dragoni, L. (2005). Understanding the Emergence of State Goal Orientation in Organizational Work Groups: The Role of Leadership and Multilevel Climate Perceptions. Journal of Applied Psychology, 90, 1084-1095.

Dunlap, W. P., Burke, M. J. y Smith-Crowe, K. (2003). Accurate Test of Statistical Significance for rwg and AD Interrater Agreement Indices. Journal of Applied Psychology, 88, 356-362.

Ferris, G. R., Arthur, M. A., Berkson, H. M., Kaplan, D. M., Harrell-Cook, G. y Frink, D. D. (1998). Toward a Social Context Theory of Human Resource Management- Organizational Effectiveness Relationship. Human Resource Management Review, 8, 235-264.

Ferris, G. R. y Kacmar, K. M. (1992). Perceptions in Organizational Politics. Journal of Management, 18, 93-116.

Glick, W. H. (1985). Conceptualizing and Measuring Organizational and Psychological Climate: Pitfalls in Multilevel Research. Academy of Management Review, 10, 601-616.

Goldstein, H. (2003). Multilevel Statistical Models. New York, NY: Halstead Press.

González-Romá, V. (2011). El Clima de los Equipos de Trabajo: Una Propiedad Configuracional. Papeles del Psicólogo, 32, 48-58.

González-Romá, V., Peiró, J. M., Subirats, M. y Mañas, M. A. (2000). The Validity of Affective Work Team Climates. En M. Vartiainen, F. Avallone y N. Anderson (Eds.), Innovative Theories, Tools, and Practices in Work and Organizational Psychology (pp.97-110). Ashland, OH: Hogrefe \& Huber Publishers.

González-Romá, V., Tomás, I. y Ferreres, A. (1995). Análisis de las Propiedades Psicométricas del Cuestionario de Clima organizacional FOCUS93 en una Muestra Multiprofesional. Revista de Psicología del Trabajo y de las Organizaciones, 11, 5-18.

Hellriegel, D. y Slocum, J. W. Jr. (1974). Organizational Climate: Measures, Research and Contingencies. Academy of Management Journal, 17, 255-280.

Howe, J. G. (1977). Group Climate: An Exploratory Analysis of Construct Validity. Organizational Behavior and Human Performance, 19, 106-125.

Jackofski, E. F. y Slocum, J. W. Jr. (1988). A Longitudinal Study of Climates. Journal of Organizational Behavior, 9, 319-334.

James, L. R. (1982). Aggregation Bias in Estimates of Perceptual Agreement. Journal of Applied Psychology, 67, 219-229.

James, L. R., Choi, C. C., Ko, C. H. C., McNeil, P. K., Minton, M. K., Wright, M. A. y Kim, K. (2008). Organizational and Psychological Climate: A review of Theory and Research. European Journal of Work and Organizational Psychology, 17, 5-32.

James, L. R., Demaree, R. G. y Wolf, G. (1984). Estimating Within-Group Interrater Reliability with and without Response Bias. Journal of Applied Psychology, 69, 85-98.

James, L. R. y Jones, A. P. (1974). Organizational Climate: A Review of Theory and Research. Psychological Bulletin, 81, 1096-1112.

Judge, T. A., Hulin, C. L. y Dalal, R. S. (2012).Job Satisfaction and Job Affect. En S.W.J. Kozlowski (Ed.), The Oxford Handbook of Organizational Psychology. New York, NY: Oxford University Press.

Judge, T. A. y Klinger, R. (2007). Job Satisfaction. Subjective Well-Being at Work. En M. Eid y R. Larsen (Eds), The Science of Subjective Well-Being. New York, NY: Guilford Publications.
Judge, T. A. y Piccolo, R. F. (2004). Transformational and Transactional Leadership: A Meta-Analytic Test of their relative Validity. Journal of Applied Psychology, 89, 755-768.

Judge, T. A., Thoresen, C. J., Bono, J. E. y Patton, G. K. (2001). The Job Satisfaction-Job Performance Relationship: A Qualitative and Quantitative Review. Psychological Bulletin, 127, 376-407.

Klein, K. J., Conn, A. B., Smith, D. B. y Sorra, J. S. (2001). Is Everyone in Agreement? An Exploration of Within-Group Agreement in Employee Perceptions of the Work Environment. Journal of Applied Psychology, 86, 3-16.

Klein, K. J., Bliese, P. D., Kozlowski, S. W. J., Dansereau, F., Gavin, M.B., Griffin, M.A.,.....Bligh, M.C. (2000). Multilevel Analytical Techniques: Commonalities, Differences, and Continuing Questions. En S.W.J. Kozlowski y K.J. Klein (Eds), Multilevel Theory, Research and Methods in Organizations (pp.512-553). San Francisco, CA: Jossey-Bass.

Klein, K. J., Dansereau, F. y Hall, R. J. (1994). Level Issues in Theory Development, Data Collection, and Analysis. Academy of Management Review, 19, 195-229.

Kopelman, R. E., Brief, A. P. y Guzzo, R. A. (1990). The Role of Climate and Culture in Productivity. En B. Schneider (Ed.), Organizational Climate and Culture (pp.282-318). San Francisco, CA: Jossey-Bass.

Kozlowski, S. W. J. y Klein, K. J. (2000). Multilevel Theory, Research and Methods in Organizations. San Francisco, CA: Jossey-Bass.

Kuenzi, M. y Schminke, M. (2009). Assembling Fragments into a Lens: A Review, Critique and Proposed Research Agenda for the Organizational Work Climate Literature. Journal of Management, 35, 634-717.

LeBreton, J. M. y Senter, J. L. (2008). Answers to Twenty Questions about Interrater Reliability and Interrater Agreement. Organizational Research Methods, 11, 815-852.

Lewin, K., Lippit, R. y White, R. K. (1939). Patterns of Aggressive Behaviour in Experimental Created "Social Climates". Journal of Social Psychology, 10, 271-299.

Lloret, S., González-Romá, V. y Peiró, J. M. (1993). El Cuestionario CSLPSEAP/33. Un Estudio acerca de su Validez. Psicológica, 14, 21-30.

Locke, E. A. (1976). The Nature and Causes of Job Satisfaction. En M. D. Dunnette (Ed), Handbook of Industrial and Organizational Psychology (pp.1297-1349). Chicago, IL: Rand McNally.

Luthans, F., Norman, S. M., Avolio, B. J. y Avey, J. B. (2008). Supportive Climate and Organizational Success: The Mediating Role of Psychological Capital. Journal of Organizational Behavior, 29, 219-238.

McCullag, P. y Nelder, J. A. (1989). Generalized to Line Models. New York, NY: Chapman \& Hall.

Mañas, M. A., González-Romá, V. y Peiró, J. M. (1999). El Clima de los Equipos de Trabajo: Determinantes y Consecuencias. Almería: I.E.A.-UAL.

Mañas, M.A., González-Romá, V., Peiró, J.M. y Lloret, S. (2003). Psychological Climate and Attitudinal Job Outcomes: A Test of the Reciprocal Influence Hypothesis. Revista de Psicología Social Aplicada, 13, 45-60.

Martinez-Tur, V., Tordera, N., Peiró, J. M. y Potocnik, K. (2011). Linking Service Climate and Disconformation of Expectations as Predictors of Customer Satisfaction: A Cross-Level Study. Journal of Applied Social Psychology, 41, 1189-1213.

Medina, F. J., Munduate, L., Martínez, I., Dorado, M. A. y Mañas, M. A. (2004). Efectos Positivos de la Activación del Conflicto de Tarea sobre el Clima de los Equipos de Trabajo. Revista de Psicología Social, 19, 3-15.

Morgeson F. P. y Hoffman, D. A. (1999). The Structure and Function of Collective Constructs: Implications for Multilevel Research and Theory Development. Academy of Management Journal, 24, 249-265.

Organ, D.W. (1988). Organizational Citizenship Behavior: The Good Soldier Syndrome. Lexington, MA: Lexington Books.

Organ, D. W. y Ryan, K. (1995). A Meta-Analytic Review of Attitudinal and Dispositional Predictors of Organizational Citizenship Behavior. Personnel Psychology, 48, 775-802.

Ostroff, C. (1992). The Relationship between Satisfaction, Attitudes and Performance: An Organizational Level Analysis. Journal of Applied Psychology, 78, 569-582.

Ostroff, C. (1993). The Effects of Climate and Personal Influences on Individual Behavior and Attitudes in Organizations. Organizational Behavior and Human Performance, 56, 56-90.

Ostroff, C. y Bowen, D. E. (2000). Moving HR to higher level: HR practices and organizational effectiveness. En K.J. Klein y S.W.J. Kozlowski 
(Eds.), Multilevel theory, research and methods in organizations (pp.211-266). San Francisco, CA: Jossey-Bass.

Ostroff, C., Kinicki, A. y Tamkins, M. (2003). Organizational Culture and Climate. In W.C. Borman y D.R. Ilgen (Eds.), Handbook of psychology: Industrial and organizational psychology, vol.12 (pp.565-593). New York, NY: John Wiley \& Sons, Inc.

Pardo, A., Ruiz, M. A. y San Martin, R. (2007). Cómo Ajustar e Interpretar Modelos Multinivel con SPSS. Psicothema, 19, 308-321.

Parker, C. P., Baltes, B. B., Young, S.A., Huff, J.W., Altmann, R.A., Lacost, H.A. y Roberts, J.E. (2003). Relationships between Psychological Climate Perceptions and Work Outcomes: A Meta-Analytic Review. Journal of Organizational Behavior, 24, 389-416

Patterson, M., Warr, P. y West, M. (2004). Organizational Climate and Company Productivity: The Role of Employee Affect and Employee Level. Journal of Occupational and Organizational Psychology, 77, 193-216.

Peiró, J. M. (1984). Psicología de la Organización. Madrid : UNED.

Pirola-Merlo, A., Hartel, C., Mann, L. y Hirst, G. (2002). How Laders Influence the Impact of Affective Events on Team Climate and Performance in R\&D Teams. The Leadership Quarterly, 13, 561-581.

Raudenbush, S. W. y Brik, A.S. (2002). Hierarchical Linear Models: Applications and Data Analysis Methods. Thousand Oaks, CA: Sage.

Reichers,, A. E. y Schneider, B. (1990). Climate and Culture: An Evolution of Constructs. En B. Schneider (Ed.), Organizational Climate and Culture (pp.5-39). San Francisco, CA: Jossey-Bass.

Salancik, G.R. y Pfeffer, J. (1978). A Social Information Processing Approach to Job Attitudes and Task Design. Administrative Science Quarterly, 23, 224-253.

Salanova, M., Agut, S. y Peiró, J. M. (2005). Linking Organizational Resources and Work Engagement to Employee Performance and Customer Loyalty: The Mediation of Service Climate. Journal of Applied Psychology, 90, 1217-1227.

Salanova, M., Llorens, S., Cifre, E. y Martínez, I. M. (2012). We Need a Hero! Toward a Validation of the Healthy and Resilient Organization (HERO) Model. Group and Organization Management, 37, 785-822.

Schein, E. H. (2004). Organizational Culture and Leadership. San Francisco, CA.: Jossey-Bass.

Schneider, B. (1975). Organizational Climate: An Essay. Personnel Psychology, 28, 447-479.

Schneider, B. (1990). Organizational Climate and Culture. San Francisco, CA: Jossey-Bass.

Schneider, B. (2000). The Psychological Life of Organizations. En N. M. Ashkanasy, C. P. M. Wilderom y M. F. Peterson (Eds.), Handbook of Organizational Culture and Climate (pp. xvii-xxi). Thousands Oaks, CA: Sage Publications.

Schneider, B. y Barlett, C. J. (1968). Individual Differences and Organizational Climate, I: The Research Plan and Questionnaire Development. Personnel Psychology, 21, 323-333.

Schneider, B., Ehrhart, M. G. y Macey, W. H. (2011). Perspectives on Organizational Climate and Culture. En S.E. Zedeck (Ed). Handbook of Psychology (pp.373-414). Washington, DC: APA.

Schneider, B., Ehrhart, M. G. y Macey, W. H. (2013). Organizational Climate and Culture. Annual Review of Psychology, 64, 361-388.

Schneider, B. y Reichers, A. E. (1983). On the Ethiology of Climates. Personnel Psychology, 36, 19-39.
Schneider, B., Salvaggio, A. N. y Subirats, M. (2002). Climate Strength: A New Direction for Climate Research. Journal of Applied Psychology, 87, 220-229.

Schneider, B., White, S. y Paul, M. (1998). Linking Service Climate and Customers Perceptions of Service Quality: Test of a Causal Model. Journal of Applied Psychology, 83, 150-163.

Schulte, M., Ostroff, C. y Kinicki, A. J. (2006). Organizacional Climate Systems and Psychological Climate Perceptions: A Cross-Level Study of Climate-Satisfaction Relationships. Journal of Occupational and Organizational Psychology, 79, 645-671.

Schulte, M., Ostroff, C., Shmulyian, S. y Kinicki, A. (2009). Organizational Climate Configurations: Relationships to Collective Attitudes, Customer Satisfaction, and Financial Performance. Journal of Applied Psychology, 94, 618-634

Shrout, P. E. y Fleiss, J. L. (1979). Intraclass Correlations: Uses in Assessing Rater Reliability. Psychological Bulletin, 86, 420-428.

Solé, F. y Llinás-Audet, (2011). De la Burocracia Profesional a la Tecnópolis: Los Desafíos Estratégicos de la Gestión Universitaria. Revista de Educación, 355, 17-30.

Sora, B., Caballer, A., Peiró, J. M. y de Witte, H. (2009). Job Insecurity Climate's on Employees' Job Attitudes: Evidence from two European Countries. European Journal of Work and Organizational Psychology, 18, 125 147.

Van de Vorde, K., Van Veldhoven, M. y Paauwe, J. (2012). Relationships between Work Unit Climate and Labour Productivity in the Financial Sector: A Longitudinal Test of the Mediating Role of Work Satisfaction. European Journal of Work and Organizational Psychology, 23, 295-309.

Van Muijen, J. J., Koopman, P., De Witte, K., De Cock, G., Susanj, Z., Lemoine, C., .... Turnipseed, D. (1999). Organizational Culture: The Focus Questionnaire. European Journal of Work and Organizational Psycholoy, 8, 551-568.

Vigoda, E. (1999). Organizational Politics, Job Attitudes and Work Outcomes: Exploration and Implications for the Public Sector. Journal of Vocational Behavior, 57, 326-347.

Vigoda, E. (2002). Stress-Related Aftermaths to Workplace Politics: the Relationships among Politics, Job Distress and Aggressive Behavior in Organizations. Journal of Organizational Behavior, 23, 571-591.

Vigoda-Gadot, E. y Beeri, I. (2012). Change-Oriented Organizational Citizenship Behavior in Public Administration: The Power of Leadership and the Cost of Organizational Politics. Journal of Public Administration Research, 22, 573-596.

Weiss, H. M. (2002). Deconstructing Job Satisfaction Separating Evaluations, Beliefs and Affective Experiences. Human Resource Management Review, 2, 173-194.

Wright, T. A. (2005). The Role of "Happiness" in Organizational Research: Past, Present and Future Directions. En P. L. Perrewe y D. C. Ganster (Eds.), Research in Occupational Stress and Well-being, 4, (p. 221-264). Amsterdam: JAI Press.

Wright, T. A. (2006). The Emergence of Job Satisfaction in Organizational Behavior: A Historical overview of the dawn of Job Attitude Research. Journal of Management History, 12, 262-277.

(Articulo recibido: 22-03-2013; revisado: 17-07-2013; aceptado: 03-03-2014) 